\title{
Tinnitus suppression by electric stimulation of the auditory nerve
}

\author{
Janice E. Chang ${ }^{1 *}$ and Fan-Gang Zeng ${ }^{1,2 *}$ \\ ${ }^{1}$ Department of Anatomy and Neurobiology, University of California Irvine, Irvine, CA, USA \\ ${ }^{2}$ Department of Otolaryngology - Head and Neck Surgery, University of California Irvine, Orange, CA, USA
}

\author{
Edited by: \\ Jos J Eggermont, University of \\ Calgary, Canada \\ Reviewed by: \\ David Baguley, Cambridge \\ University Hospital, UK \\ Katrien Vermeire, University of \\ Innsbruck, Austria \\ Andreas Buechner, Medical \\ University of Hannover, Germany \\ *Correspondence: \\ Janice E. Chang and Fan-Gang \\ Zeng, Department of Anatomy and \\ Neurobiology, Hearing and Speech \\ Lab, University of California, Irvine, \\ 110 Medical Science E, Irvine, CA \\ 92697, USA. \\ e-mail: janicec@uci.edu, \\ fzeng@uci.edu
}

Electric stimulation of the auditory nerve via a cochlear implant $(\mathrm{Cl})$ has been observed to suppress tinnitus, but parameters of an effective electric stimulus remain unexplored. Here we used $\mathrm{Cl}$ research processors to systematically vary pulse rate, electrode place, and current amplitude of electric stimuli, and measure their effects on tinnitus loudness and stimulus loudness as a function of stimulus duration. Thirteen tinnitus subjects who used Cls were tested, with nine (70\%) being "Responders" who achieved greater than $30 \%$ tinnitus loudness reduction in response to at least one stimulation condition and the remaining four (30\%) being "Non-Responders" who had less than 30\% tinnitus loudness reduction in response to any stimulus condition tested. Despite large individual variability, several interesting observations were made between stimulation parameters, tinnitus characteristics, and tinnitus suppression. If a subject's tinnitus was suppressed by one stimulus, then it was more likely to be suppressed by another stimulus. If the tinnitus contained a "pulsating" component, then it would be more likely suppressed by a given combination of stimulus parameters than tinnitus without these components. There was also a disassociation between the subjects' clinical speech processor and our research processor in terms of their effectiveness in tinnitus suppression. Finally, an interesting dichotomy was observed between loudness adaptation to electric stimuli and their effects on tinnitus loudness, with the Responders exhibiting higher degrees of loudness adaptation than the Non-Responders. Although the mechanisms underlying these observations remain to be resolved, their clinical implications are clear. When using a $\mathrm{Cl}$ to manage tinnitus, the clinical processor that is optimized for speech perception needs to be customized for optimal tinnitus suppression.

Keywords: tinnitus, cochlear implant, loudness adaptation, electric stimulation

\section{INTRODUCTION}

Tinnitus is an auditory disorder known as "ringing of the ears or head" which affects 50 million Americans and an estimated 600 million worldwide, according to the American Tinnitus Association (ATA; www.ata.org). Its severity can range from being temporary and unobtrusive to debilitating and life-impairing. Tinnitus has steadily increased by $18 \%$ per year since 2001 in soldiers returning from the Global War on Terror, and is currently the number one cause of service-connected disability (ATA, 2011). Although management treatments and therapies are available, there currently exists no cure (Goodey, 2007).

While neural mechanisms causing tinnitus and hearing loss are not identical, the incidence of tinnitus is highly correlated with, and believed to be related to hearing loss (Axelsson and Ringdahl, 1989). A study by the National Study of Hearing showed that hearing impairment is the dominant factor in predicting the occurrence of prolonged spontaneous tinnitus (Coles et al., 1988; Tyler, 2000). Although not all individuals experiencing tinnitus have hearing loss, those who have a hearing loss experience an $83 \%$ higher risk of developing tinnitus over those who do not have a hearing loss (Nondahl et al., 2002). Interestingly, when these individuals are fit with hearing aids, approximately half of hearing aid users with tinnitus report that their amplification also provides either partial or total relief from their tinnitus (Surr et al., 1985).

Individuals with more severe hearing loss may receive a cochlear implant (CI) to help restore hearing (Zeng, 2004). While most individuals who have tinnitus are not profoundly deaf (Tyler, 2000), a reported $66-86 \%$ of CI users indeed experience tinnitus (Tyler and Kelsay, 1990; Hazell et al., 1995; Miyamoto et al., 1997; Quaranta et al., 2004; Bovo et al., 2010). Evidence of cochlear electrical stimulation has been noted to benefit tinnitus, and CIs have been suggested as a potential therapeutic since they became commercially available in the early 1980s (House and Brackmann, 1981). In recent years, the benefit of CI on tinnitus has been widely reported in many studies. Efficacy rates range from 34 to 93\% (Tyler and Kelsay, 1990; Miyamoto et al., 1997; Pan et al., 2009; Bovo et al., 2010). On the other hand, a smaller percentage of $16.7-41.5 \%$ of CI users reports no effect of the CI on their tinnitus (Tyler and Kelsay, 1990; Miyamoto et al., 1997; Bovo et al., 2010).

Significantly, most studies investigating CI effects on tinnitus have used standard multichannel CIs using speech processors optimized for speech. Observational questionnaires comparing 
pre- and post-implantation tinnitus have found reduction in tinnitus intensity or loudness (Ito and Sakakihara, 1994; Miyamoto et al., 1997; Ruckenstein et al., 2001), decreased annoyance, and general reports that "the majority of patients thought that their CI was helpful in tinnitus suppression" (Souliere et al., 1992). Changes in tinnitus pitch and timber following implantation have also been noted (Souliere et al., 1992; Miyamoto et al., 1997). These effects have been attributed to surgical insertion of the intra-cochlear electrode (Baguley and Atlas, 2007) as well as plastic changes in the auditory system brought about by $\mathrm{CI}$ use (Quaranta et al., 2004; Baguley and Atlas, 2007). Of note, current candidacy requirements for CI implantation are strictly based on hearing capabilities, as measured by severe-to-profound sensorineural hearing loss that is not substantially improved by hearing aids, and speech recognition test scores in the United States, Canada, and the United Kingdom (UK Cochlear Implant Study Group, 2004; Balkany et al., 2007; Amoodi et al., 2012). Because clinical processors used in CIs are designed to improve speech perception (Wilson et al., 1991; Zeng et al., 2008), their effects on tinnitus are usually considered to be secondary.

There is limited literature on optimizing electric stimulation for tinnitus suppression. Rubinstein et al. (1999) used high-rate stimulation to produce a pattern of spontaneous-like firing similar to that seen in the healthy auditory nerve, which is thought to represent the auditory "code for silence." He tested the effectiveness of high-rate stimulation [4800 pulses per second (pps)] in three Cochlear CI users with tinnitus and found that one subject showed level-dependent tinnitus suppression with complete adaptation to the electric stimulus, one subject showed tinnitus suppression only in the presence of a stimulus percept, and one subject reported no change in tinnitus at her maximal comfort level of stimulation (Rubinstein et al., 2003). On the other hand, Dauman et al. (1993) explored low-rate bipolar stimulation in two Cochlear CI patients, finding that 125 pps stimuli was the most effective in that it required the lowest amount of current to achieve suppression, and that the effectiveness of stimulation could vary by place. A parametric study exploring various combinations of stimuli parameters also found low-rate stimuli effective at completely suppressing tinnitus in a single Advanced Bionics HiRes CI user (Zeng et al., 2011). In this study we sought to corroborate these findings in a larger subject size using various CI devices. The goal of the present study is twofold: (1) to use flexible research processors to systematically vary electric stimulation parameters and measure their effects on tinnitus suppression, and (2) to identify relationships between tinnitus characteristics and electric stimulation to tinnitus suppression.

\section{MATERIALS AND METHODS SUBJECTS}

Subjects who had chronic tinnitus and a CI were screened for the study. Prior to enrollment, subjects completed an online tinnitus survey, including a questionnaire about their hearing loss and tinnitus, the Beck Depression Inventory (BDI), Beck Anxiety Inventory, Tinnitus Handicap Index, and Tinnitus Severity Index. They also provided audiological records, which included their speech recognition scores as assessed by the standardized Hearing in Noise Test (HINT; Nilsson et al., 1994). Subjects who had not been evaluated by a physician for their tinnitus, had a treatable type of tinnitus, or were on medications or other treatments for their tinnitus were excluded from the study. All subjects perceived their tinnitus for a minimum of six months.

Thirteen CI users with chronic tinnitus, 11 female and two male of average age $60.8 \pm 13.6$ years (mean \pm SD) participated in the study (Table 1). Etiology of hearing loss varied, and all subjects had severe to profound hearing loss in the non-implanted ear except for S11, who had sudden sensorineural hearing loss in one ear and normal hearing in the other. Average duration of hearing loss was $27.9 \pm 11.7$ years and subjects had an average

Table 1 | Patient demographics.

\begin{tabular}{|c|c|c|c|c|c|c|c|}
\hline & \multirow[t]{2}{*}{ Gender } & \multirow[t]{2}{*}{ Age } & \multicolumn{2}{|c|}{ Hearing loss } & \multicolumn{3}{|c|}{ Cochlear implant (CI) } \\
\hline & & & Etiology & Duration (years) & Cl use (years) & $\mathrm{Cl}$ side & HINT sentence ( $\%$ correct) \\
\hline S1 & $\mathrm{F}$ & 59 & Otosclerosis & 26 & 1 & $L$ & 76 \\
\hline S2 & $\mathrm{F}$ & 67 & Genetic & 34 & 6 & $\mathrm{R}$ & 100 \\
\hline S3 & $\mathrm{F}$ & 74 & Sensorineural & 28 & 9 & $L$ & 97 \\
\hline S4 & $\mathrm{F}$ & 77 & Autoimmune & 21 & 4 & $L$ & 75 \\
\hline \multirow[t]{2}{*}{ S5 } & $\mathrm{F}$ & 50 & Autoimmune & 31 & 13 & Both & $96(\mathrm{~L})$ \\
\hline & & & & & & & $63(R)$ \\
\hline S6 & $\mathrm{F}$ & 61 & Meniere's & 29 & 2 & $L$ & 96 \\
\hline \multirow[t]{2}{*}{ S7 } & $\mathrm{F}$ & 72 & Idiopathic & 15 & 9 & Both & n.a. (L) \\
\hline & & & & & & & $87(\mathrm{R})$ \\
\hline S8 & $\mathrm{F}$ & 59 & Sensorineural & 43 & 8 & $L$ & 44 \\
\hline S9 & M & 86 & Noise-induced & 39 & 3 & $\mathrm{R}$ & 8 \\
\hline S10 & $\mathrm{F}$ & 43 & Radiation-induced & 14 & 1 & $L$ & 76 \\
\hline S11 & $\mathrm{M}$ & 50 & Idiopathic & 5 & 4 & $\mathrm{R}$ & 70 \\
\hline S12 & $\mathrm{F}$ & 45 & Congenital rubella & 45 & 6 & $\mathrm{~L}$ & 0 \\
\hline S13 & $\mathrm{F}$ & 47 & Genetic & 33 & 1 & $\mathrm{R}$ & 100 \\
\hline
\end{tabular}

n.a. = not available. 
CI use of $5.15 \pm 3.8$ years with at least one year of experience. Six subjects used Cochlear devices, four used Advanced Bionics, and three used Med-El. These CI users had sentence recognition ranged from 0 to $100 \%$, and covered the full range from poor to good performers (Nilsson et al., 1994; Friesen et al., 2001).

\section{TINNITUS CHARACTERISTICS}

Patient tinnitus characteristics are shown in Table 2. Prior to testing, patients were asked if "their tinnitus has improved since cochlear implantation." Seven (54\%) patients reported "Yes" or "sometimes," while five subjects (38\%) reported "No" and one subject was unsure. All patients had experienced tinnitus for a minimum of one year, ranging from 1 to 49 years with an average of $20.5 \pm 15.3$ years. Tinnitus Handicap Inventory (THI) scores showed $38 \%$ of subjects (5 of 13 ) with moderate handicap, 31\% (4 of 13) with mild handicap, and 31\% (4 of 13) with slight or no tinnitus handicap. Ninety-two percent (12 of 13) of patients had minimal depression (the lowest grade possible) based on the BDI, and one subject experienced mild depression.

Average baseline loudness rating (LR) of their tinnitus was $4.4 \pm 1.9$ on a numeric scale from $0-10$ (Table 3 ). Subjects reported a variety of different sounds descriptive of their tinnitus. The most common sound perceived was humming (85\%, 11 subjects), followed by ringing and roaring (both 77\%, 10 subjects each), then buzzing and the "inside of a seashell" (both $69 \%$, nine subjects each). Two subjects reported hearing music alongside their tinnitus.

\section{STIMULI AND PROCEDURE}

All stimuli were delivered to the subject's CI using a research processor, controlled by a customizable research interface connected to a computer. Use of the customizable research interfaces allowed fixed pulse trains to be continuously delivered to only a single electrode at a given stimulation rate, place, or level, as distinct from commercial speech processors. Stimuli were fixed, charge-balanced, biphasic anodic-first pulse trains, delivered with a given stimulation rate (low: 100 or 200 pps and high: 5000 pps), stimulation place (apical, middle, or basal electrode) and stimulation level (corresponding to soft, medium, and loud) for a total combination of 18 stimuli conditions. Stimulated electrodes were selected as the apical- and basal-most electrodes, as well as the electrode in the middle of the array. Apical, middle, and basal electrodes were selected, respectively as electrodes 22,11 , and 1 for Cochlear devices; 1, 8, 16 for Advanced Bionics; and 1, 6, and 12 for Med-El (see Figure 1). Loudness levels were determined via subject feedback, using a LR numeric scale from 0 to $10(0=$ no sound, $10=$ very uncomfortable) as a guide. On this scale, LR 3 corresponded to "soft," LR 5 to "medium," and LR 7 to "loud but comfortable."

- For Cochlear users, electric stimuli were delivered through a programmable SPEAR3 Speech Processor (Hearworks, Pty, Melbourne, Australia) and controlled via the Woomera software (Cochlear Ltd., Sydney, Australia); source code was written in Motorola DSP563xx assembly language. Subjects adjusted the volume to the appropriate loudness using a dial on the speech processor.

- For Advanced Bionics users, electric stimuli were delivered via a research interface provided by Advanced Bionics Corporation and controlled via the BionicEar Data Collection System software (Advanced Bionics LLC; Sylmar, CA, USA).

- For Med-El users, electric stimuli were delivered via the Diagnostic Interface Box connected to the CIS-PRO + processor and controlled using the CI.Studio+ 2.0 software (Med-El Corporation, Innsbruck, Austria). The clinical mapping software was used to turn off all but one electrode, which was set at the appropriate stimulation rate. A steady state sound was then delivered directly through the processor via an audio input cable to activate that given electrode.

Table 2 | Patient tinnitus characteristics.

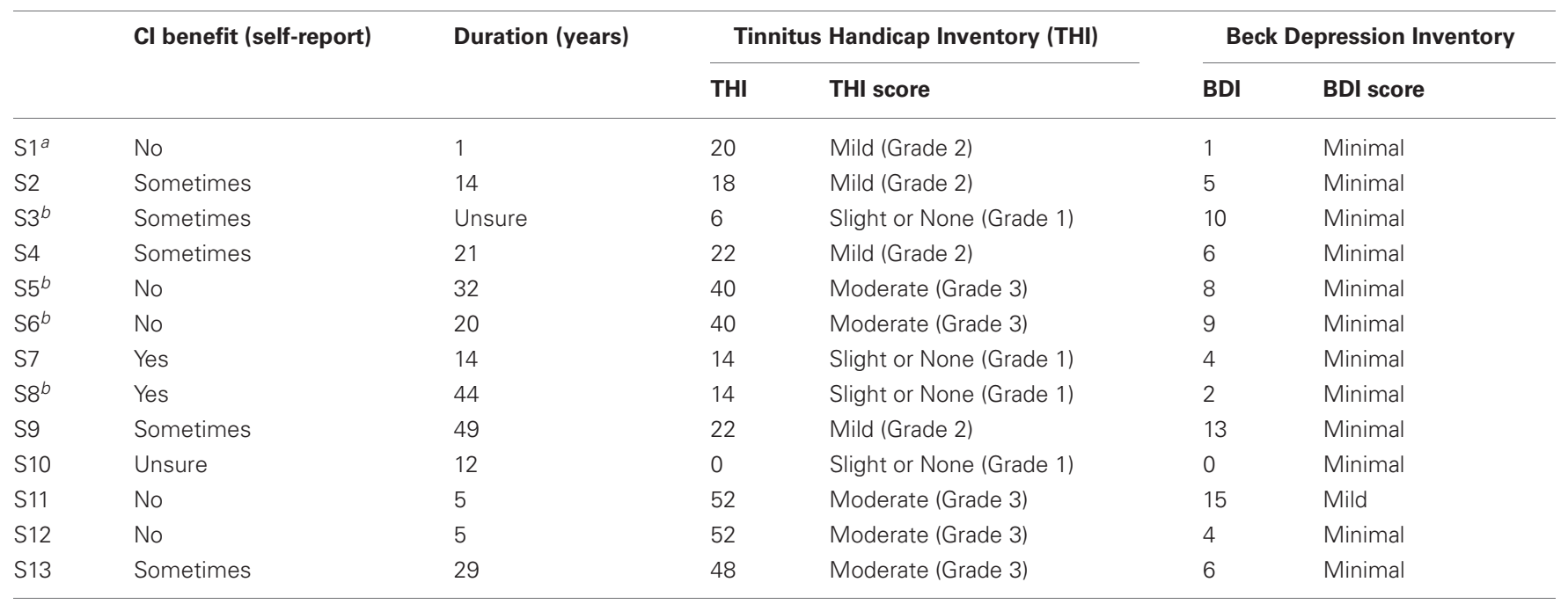

${ }^{a}$ Onset of tinnitus after implantation.

${ }^{b}$ Experienced bilateral tinnitus, with distinct sounds reported by ear. 
Table 3 | Tinnitus characteristics.

\begin{tabular}{|c|c|c|c|c|c|c|c|c|c|c|c|c|c|c|c|}
\hline & $\begin{array}{l}\text { Baseline } \\
\text { loudness }\end{array}$ & Buzz & Hum & Ring & Blow & Hiss & Roar & Whistling & Pulsating & $\begin{array}{l}\text { Constant } \\
\text { high } \\
\text { pitches }\end{array}$ & $\begin{array}{l}\text { Constant } \\
\text { low } \\
\text { pitches }\end{array}$ & $\begin{array}{l}\text { Running } \\
\text { water }\end{array}$ & $\begin{array}{l}\text { Inside of } \\
\text { a seashell }\end{array}$ & Sizzle & Other \\
\hline S2 & 3.2 & $x$ & $x$ & $x$ & & $x$ & $x$ & $x$ & $x$ & & & $x$ & $x$ & $x$ & $x^{a}$ \\
\hline \multirow[t]{2}{*}{ S3 } & $1.0(\mathrm{~L})$ & $x$ & $x$ & $x$ & & $x$ & $x$ & $x$ & & $x$ & $x$ & & $x$ & $x$ & \\
\hline & $5.0(\mathrm{R})$ & & & & & & & & & & & & & & \\
\hline \multirow[t]{2}{*}{ S5 } & $8.3(\mathrm{~L})$ & $x$ & $x$ & $x$ & & & $x$ & & & $x$ & $x$ & & $x$ & & $x$ \\
\hline & $7.3(\mathrm{R})$ & & & & & & & & & & & & & & \\
\hline \multirow[t]{2}{*}{ S6 } & $4.5(\mathrm{~L})$ & $x$ & $x$ & $x$ & & & $x$ & & $x$ & & & & $x$ & & $x$ \\
\hline & $4.9(\mathrm{R})$ & & & & & & & & & & & & & & \\
\hline S7 & 2.4 & $x$ & $x$ & & & $x$ & & & & & & & $x$ & & \\
\hline $\mathrm{S} 11$ & 5.2 & $x$ & $x$ & $x$ & & $x$ & $x$ & $x$ & $x$ & $x$ & $x$ & $x$ & $x$ & $x$ & $x$ \\
\hline $\mathrm{S} 12$ & 3.9 & & $x$ & $x$ & & & $x$ & & & & $x$ & $x$ & & & \\
\hline $\mathrm{S} 13$ & 6.0 & $x$ & $x$ & $x$ & & $x$ & $x$ & & $x$ & $x$ & & $x$ & $x$ & & $x$ \\
\hline$\#$ & $4.4 \pm 1.9$ & 9 & 11 & 10 & 2 & 6 & 10 & 5 & 5 & 7 & 7 & 5 & 9 & 3 & 8 \\
\hline$\%$ & & 69 & 85 & 77 & 15 & 46 & 77 & 38 & 38 & 54 & 54 & 38 & 69 & 23 & 62 \\
\hline
\end{tabular}

${ }^{a} S 2$ and $S 4$ reported hearing music.

\section{Apical Middle Basal

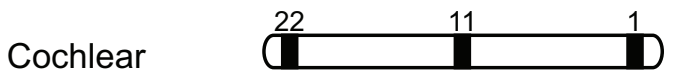

Advanced Bionics

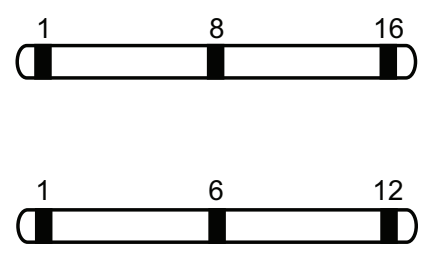

FIGURE 1 | Electrode stimulation site by condition and $\mathrm{Cl}$ manufacturer. Diagrammatic representations of electrode arrays are presented by $\mathrm{Cl}$ manufacturer, with the stimulated electrode number listed above the appropriate electrode (shaded in black) for apical (left), middle (center), and basal (right) stimulation conditions.

Prior to each trial, baseline tinnitus was assessed as the LR of the tinnitus prior to delivery of the test stimulus. Fixed, unmodulated pulse trains were then delivered to a single electrode at a fixed stimulation level for $6 \mathrm{~min}$. LRs of the tinnitus and the electric stimuli were each reported by the subject every $30 \mathrm{~s}$ for the duration of the stimuli. Tinnitus was allowed to return to baseline prior to the next testing condition. S10 had a dead region in the basal region of her cochlea secondary to radiation for a cerebellar tumor (Moore et al., 2000); S3, S6, S7, and S9 did not complete testing conditions. Testing conditions were presented in a randomized order. Due to thorough evaluation of stimulation conditions and time required between trials, total testing time could span two full days for each subject.

All patients gave informed, written consent and protocols were approved by the Institutional Review Board at the University of California Irvine.

\section{ANALYSIS}

LRs for tinnitus and electric stimuli were reported as loudness adaptation percentages, calculated as such:

$$
\text { Loudness adaptation percentage }=\frac{\mathrm{L}_{t}-\mathrm{L}_{0}}{\mathrm{~L}_{0}} \times 100,
$$

where $\mathrm{L}_{t}$ is $\mathrm{LR}$ at time $t$ and $\mathrm{L}_{0}$ is the initial LR at time $t=0$. A value of $-100 \%$ means that the tinnitus or sound is inaudible $\left(\mathrm{L}_{t}=0\right)$, while $0 \%$ means that the tinnitus or sound is unchanged from the baseline $\left(\mathrm{L}_{t}=\mathrm{L}_{0}\right)$. The adaptation data were fitted using the following equation:

$$
y(t)=s\left(1-e^{-t / \tau}\right),
$$

where $s$ represents the plateaued adaptation percentage, calculated as the average of the final five loudness estimates in terms of percentage of the original loudness at the onset of stimulation. $\tau$ represents the time constant at which the loudness percept adapted. For cases where no adaptation occurred, both $s$ (plateaued adaptation percentage) and $\tau$ (time constant) were set to 0 .

Subjects were also classified based on their responses to the tested stimuli. Tinnitus suppression outcome were based on a $30 \%$ cutoff; a tinnitus suppression of $30 \%$ or more was considered 
"successful" while suppression of less than 30\% was considered as "no suppression." Thus, subjects tested in this paradigm fit into one of two categories: "Responder" subjects, whose tinnitus adapted to at least one condition by $30 \%$ or more, or "NonResponder" subjects, whose tinnitus remained uninfluenced by electric stimulation.

Statistical analyses were performed for all subjects. Data were analyzed using Generalized Estimating Equations to control for repeated measures, using a linear scale response outcome of the minimum tinnitus adaptation percentage, or plateaued adaptation and time constant of the curve-fit variables for adaptation of the electric stimulus (SPSS/PASW Statistics 18; Somers, NY, USA).

Another Generalized Estimating Equations model using binary logistic regression was used to evaluate effects of stimulation parameters on tinnitus suppression outcome of greater or less than $-30 \%$. All regression models were built using rate, place and level as main effects; two-way interactions were consequently assessed. The statistical significance of each regression coefficient was determined using Wald Chi-square analyses, and the model was reduced by backward elimination. Variables of clinical interest were evaluated in the final model, and post-hoc analyses were performed based on pairwise contrasts.

\section{RESULTS}

\section{TINNITUS SUPPRESSION}

Figure 2 shows representative data from three subjects (S1, S2, and S5) at a high rate (5000 pps) and an apical electrode, with the loudness of the electric stimuli at soft, medium and loud levels represented in columns. Subjects S1 and S2 (in the first two rows) show loudness adaptation of both the tinnitus (filled circles) and the electric stimuli (open triangles). Note in the first panel S1 particularly-both the tinnitus and electric stimulus adapted from a soft level (LR: 3 ) to a barely audible sensation (LR: 1) for a total adaptation of $-66.7 \%$. Subject S2 also showed loudness adaptation to both her tinnitus and the electric stimuli, with her tinnitus percept adapting completely to - 100\% (LR: 0) in the medium and loud conditions. Subject S5 is a bilateral user who showed no loudness adaptation to the tinnitus in either ear (right ear: filled circles; left ear: X's) or the electric stimuli (open triangles).

Figure 3 shows the spread of suppression across tinnitus subjects, with each circle representing a single trial condition. Of 227 trials tested, 37\% (83 of 227) were successful conditions that elicited a tinnitus suppression of $30 \%$ or more, represented by the dotted line in Figure 3. Of these, 49\% (41/83) of the successful conditions yielded complete tinnitus suppression, where the

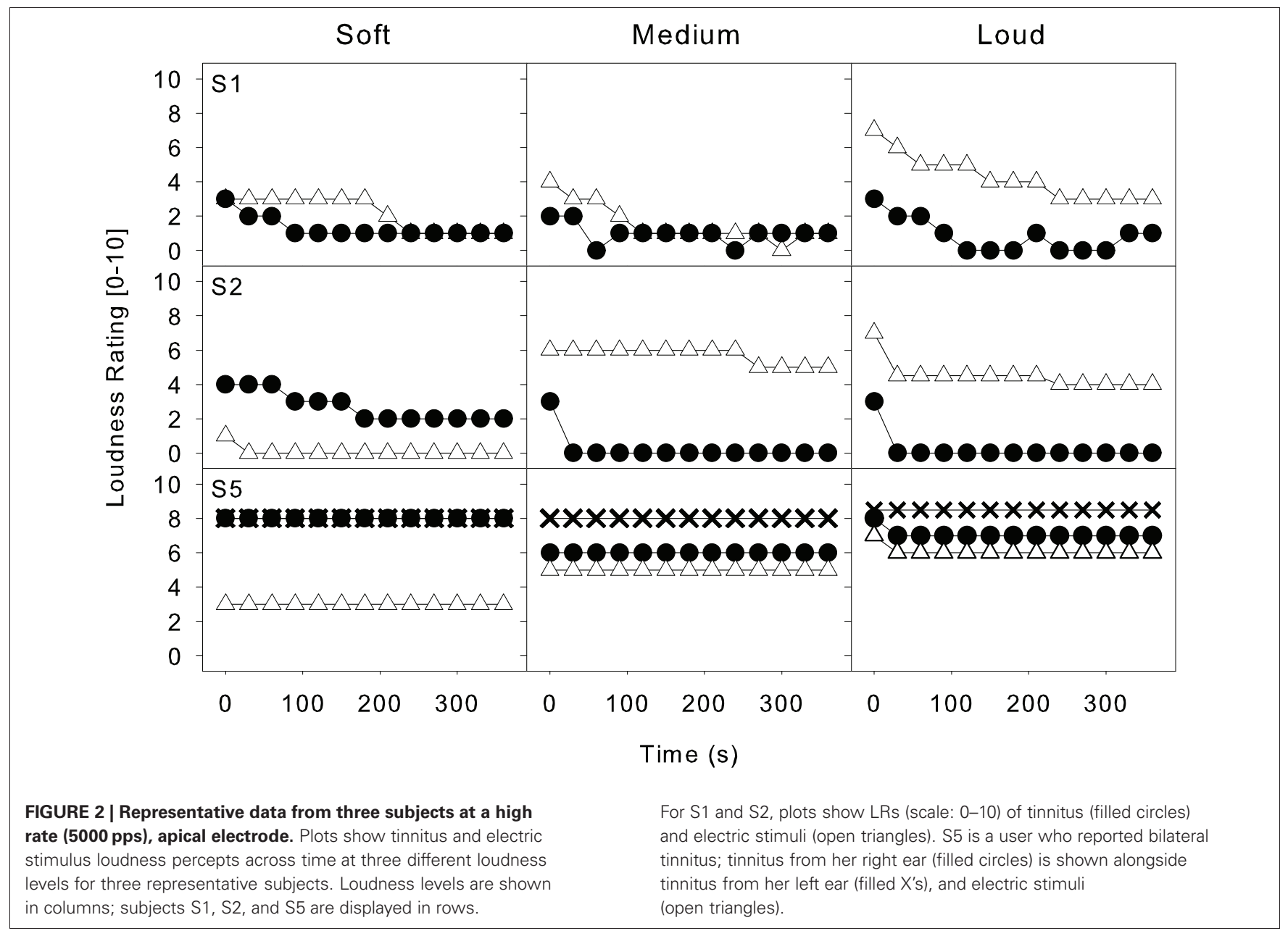






FIGURE 3 | Spread of suppression, by subject. The spread of variability in tinnitus suppression percentage across all conditions is shown for each subject. Subjects are listed across the $x$-axis and grouped as Responders (left) or Non-Responders (right). Each open circle represents the maximal tinnitus suppression for a given condition.
Bilateral tinnitus percepts for subjects S3, S5, S6, and S8 are listed by ear. Symbols at $-100 \%$ suppression (complete adaptation) were shifted by up to $5 \%$ for visualization purposes. A dotted line is shown at $-30 \%$ suppression to indicate the cutoff for successful suppression. tinnitus percept dropped to be completely imperceptible (LR: 0). Sixty-nine percent ( 9 of 13) of subjects responded to at least one condition tested: S1, S2, S3, S6, S8, S9, S10, S11, and S13. For subjects with bilateral tinnitus, ears responded uniformly: both ears responded to stimuli for S3, S6, and S8; neither ear responded for S5.

The large individual variability produced no significant effect of stimulation rate, place of stimulation, or loudness level of the electric stimulus on tinnitus suppression outcome. However, subjects who responded to one stimulus were much more likely to respond a second stimuli $\left(\chi^{2}=93.5, d f=1, p<0.001\right.$, likelihood ratio Chi-square test). Subjects who responded to at least one stimuli responded to a minimum of $27 \%$ of conditions tested (S9) and up to $78 \%$ conditions tested (S1) for an average of 50\% of successful conditions.

Figure 4 shows the percentage of successful suppression conditions based on the total number of conditions tested, with low rate stimuli represented as open bars and high rate stimuli as filled bars. A binary logistic regression model found a significant effect of loudness level on tinnitus suppression ( $p=0.049$, Wald Chisquare analysis), along with a significant rate-level interaction $(p=0.030)$. Only loud sounds were significantly more effective than soft sounds $(p=0.027)$. The significant rate-level interaction showed additionally that, for high rate sounds, medium sounds were more effective than soft sounds $(p=0.043)$ and loud sounds $(p=0.008)$. No other significant effects or interactions were found.

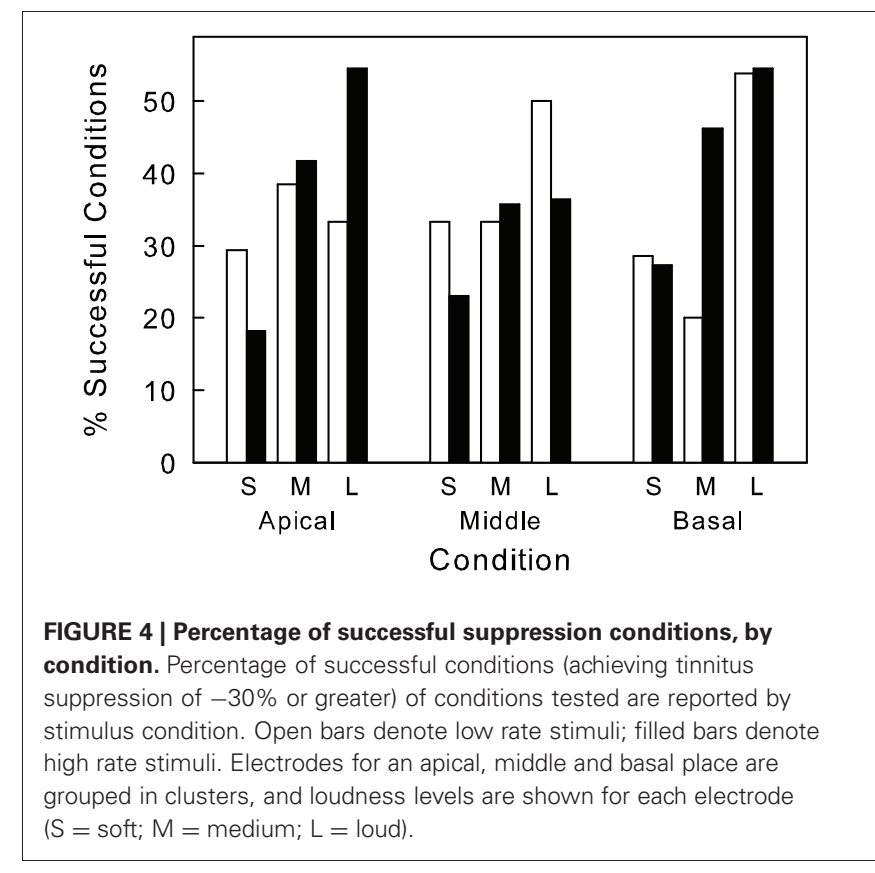

\section{RESPONDER VERSUS NON-RESPONDER SUBJECTS}

We sought to evaluate adaptation differences to external sounds (electric stimulus) as compared to internal ones (tinnitus) under the same stimulation conditions. Figure 5 (top panel) shows 


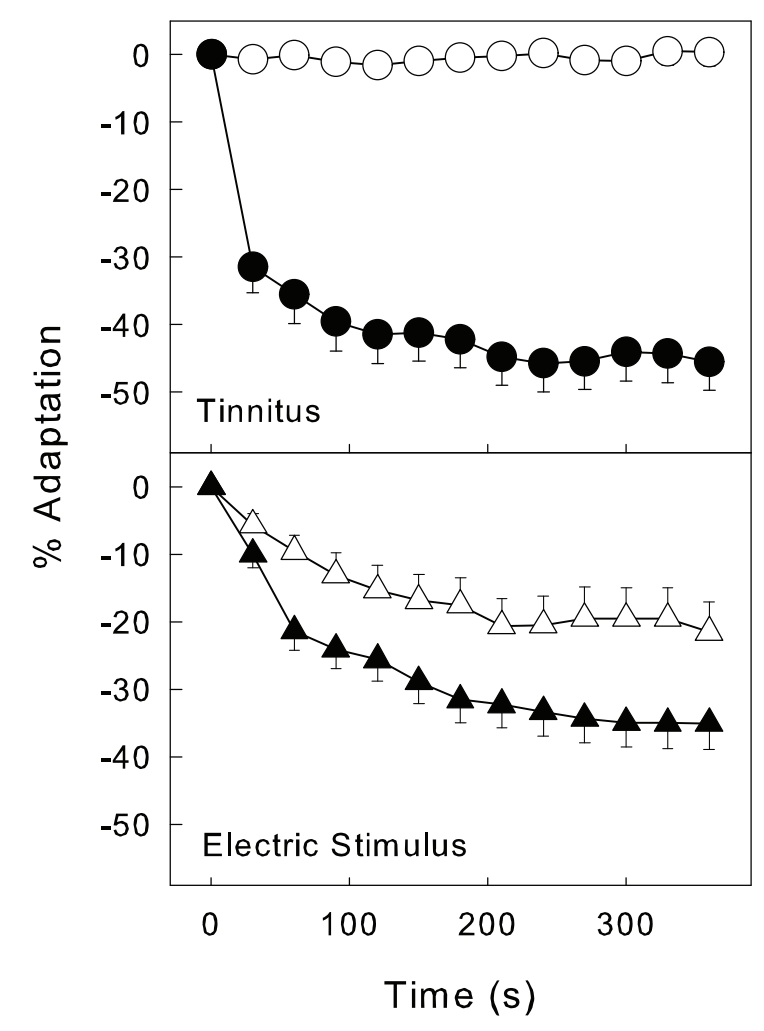

FIGURE 5 | Adaptation of tinnitus and electric stimuli, Responders versus Non-Responders. Tinnitus adaptation is shown across time in the top panel, averaged across Responder (filled circles) and Non-Responder subjects (open circles). Electric stimuli adaptation is shown across time in the bottom panel, averaged across Responder (filled triangles) and Non-Responder subjects (open triangles). Error bars indicate SEM.

a distinctive pattern of tinnitus adaptation between Responder (filled circles) and Non-Responder (open circles) subjects averaged over all conditions. No significant effects of stimulation rate, place or level were found on the overall degree of tinnitus adaptation in these subjects.

Similarly, Figure 5 (bottom panel) shows a distinctive pattern of loudness adaptation to the external electric stimulus between Responder (filled triangles) and Non-Responder (open triangles) subjects. Responders exhibited a significantly greater degree of plateaued stimulus adaptation $(-29 \%)$ than Non-Responders $(-22 \%)$, but a similar rate of loudness adaptation $\tau=0.03$ (Responders) versus $\tau=0.02$ (Non-Responders). Effects of loudness adaptation were significant at $T(225)=1.686, p=0.030$. The similar patterns between Responders and Non-Responders suggest that mechanisms of tinnitus adaptation may be related to loudness adaptation to external stimuli.

\section{DISCUSSION}

\section{COMPARISON TO PREVIOUS STUDIES}

Our findings are in line with published results reporting effectiveness of electrical stimulation of the auditory nerve via a CI to suppress tinnitus. In our study, a fixed pulse train delivered to a single electrode of the CI effectively suppressed tinnitus for
$69 \%$ of our subjects, while published efficacy rates of the CI range from 46 to $93 \%$ (reviewed by Pan et al., 2009). Two recent studies reported $80-95 \%$ of their subjects to respond positively to the CI (van de Heyning et al., 2008; Arndt et al., 2011). While our efficacy rates are not as high, our study populations are also different. These two studies focus on individuals with incapacitating unilateral tinnitus and deafness, implanted primarily for their tinnitus, while our subjects are individuals with bilateral hearing loss treated by cochlear implantation who have coincident tinnitus. By nature of our study design and patient selection, our subjects are also those whose tinnitus was resistant to complete suppression by the CI (hence seeking relief in our study). Furthermore, we found additional tinnitus benefit in four subjects who did not initially report tinnitus benefit with their own speech processor.

\section{CHARACTERIZING DIFFERENCES BETWEEN RESPONDER AND NON-RESPONDER SUBJECTS}

We evaluated demographics, hearing loss, and tinnitus profiles, and past medical histories of the two groups of subjects to identify any characteristic differences of the two groups. However, no significant differences were found between the Responder and Non-Responder groups with regard to gender, age, duration of hearing loss, duration of CI use, CI ear implant, CI device type (manufacturer), tinnitus duration, tinnitus severity (TSI), tinnitus handicap (THI), anxiety levels (BAI), or depression status (BDI). No differences were found in patient self-report of if their CI was beneficial to their tinnitus or not. No significant differences were found in general health status between the two groups either, based on self-reported past medical history of: endolymphatic shunt, vestibular neurectomy, chronic upper respiratory infection, noise exposure, sudden hearing loss, vertigo, Meniere's disease, chronic ear infections, abnormal bone growth, otorrhea, balance problems, hypertension, hypothyroidism, anemia, head tumor, autoimmune disorder(s), genetic disorder, heavy smoking, excessive caffeine use, chronic pain, insomnia, anxiety, depression, allergies, sinus congestion, or chronic infection. Interestingly, all four subjects with reported previous history of motor vehicle collision (MVC; S3, S8, S11, and S13) were Responders while none of the four Non-Responders reported having been involved in a MVC $[T(8)=2.530, p=0.035]$.

Lastly, only one of the tinnitus sound component qualities was seen more highly associated with Responder subjects: Responders were significantly more likely than Non-Responders to have pulsatile-type tinnitus, $T(8)=3.162$, $p=0.013$. No significant differences were noted for individuals with tinnitus components of: buzzing, humming, ringing, blowing, hissing, roaring, whistling, constant high pitches, constant low pitches, water-like, seashell sounds, sizzling, or any other reported sounds. Furthermore, no differences were seen between the groups either in baseline levels of tinnitus, or in the overall standard deviation (or variability) of baseline tinnitus for each subject.

\section{EFFECTIVENESS OF SOUND STIMULI BY TINNITUS QUALITY}

We also ran an analysis comparing effectiveness of each of the eighteen stimuli by the sound components of tinnitus (Table 4). No stimuli were significantly more or less effective for tinnitus with buzzing, humming, blowing, constant high pitches, constant 
low pitches, water-like, seashell sounds, or any "other" reported sound components. However, for several sounds, particular stimuli were found to be significantly more effective for subjects reporting that given tinnitus sound component, than for subjects who did not report that sound. For example, individuals reporting tinnitus with a "roaring" component achieved a significantly greater amount of tinnitus suppression with a low rate, soft stimuli to an apical electrode as compared to subjects without a roaring tinnitus component, $T(14.140)=2.853, p=0.013$; $T$-values are reported in Table 4. Ringing was the only sound that was predictive of negative predictability; namely, individuals with ringing tinnitus were less likely to respond to high rate stimuli of a soft-loudness level to either middle or basal electrodes. Tinnitus with a pulsatile component was easily suppressed with any of the six loud sound conditions except to an apical electrode with a low stimulation rate.

\section{TINNITUS AND ELECTRIC STIMULUS ADAPTATION: INSIGHTS INTO UNDERLYING MECHANISMS}

Loudness adaptation to externally presented electric stimuli in these subjects, as compared to simultaneous tinnitus adaptation, may also be insightful to mechanisms of tinnitus production. Despite a large variability in tinnitus response between subjects, a significant level-dependence was observed for tinnitus adaptation, with loud sounds achieving more tinnitus adaptation than soft sounds. While this may be partially attributed to a masking effect of electric stimuli on the tinnitus percept (Vernon, 2000; Vernon and Meikle, 2000), simple masking may not fully explain the observed results.

First, a large variability in tinnitus suppression results may indicate a heterogeneous population of tinnitus subjects. Where a final common pathway has been implicated for the perception of tinnitus (Shulman et al., 2009), evidence of tinnitus-related activity has been observed from the peripheral auditory system, through the brainstem and up to the cortex (Muhlnickel et al., 1998; Eggermont and Roberts, 2004; Roberts et al., 2010; Kaltenbach, 2011). Variability in both tinnitus characteristics

Table 4 | Effectiveness of sound stimuli by tinnitus quality.

\begin{tabular}{lll}
\hline LOW RATE & & \\
$\begin{array}{l}\text { Apical } \\
\text { Middle }\end{array}$ & Soft & Roaring: $T(14.140)=2.853, p=0.013$ \\
Basal & Loud & Pulsating: $T(12)=2.509, p=0.027$ \\
& Loud & Sizzling: $T(11)=2.279, p=0.044$ \\
& & Pulsating: $T(7.633)=3.601, p=0.008$ \\
HIGH RATE & & \\
Apical & Loud & Hissing: $T(9)=2.415, p=0.039$ \\
& & Pulsating: $T(9)=2.415, p=0.039$ \\
Middle & Loud & Hissing: $T(4.890)=3.365, p=0.021$ \\
& & Pulsating: $T(4.890)=3.365, p=0.021$ \\
& Soft & (Ringing: $T(11)=-2.589, p=0.025)$ \\
Basal & Loud & Whissing: $T(9)=4.492, p=0.002$ \\
& & Pulsating: $T(9)=2.600, p=0.029$ \\
& & Pulsating: $T(11)=2.383, p=0.002$ \\
& Medium & (Ringing: $T(9)=-8.435, p=0.000)$
\end{tabular}

described and responses to the stimuli tested here may indicate tinnitus manifesting from different sources.

Next, patterns of adaptation to electric stimuli are different from those to tinnitus adaptation. Loudness adaptation to electric stimuli showed significant effects of stimulation rate $(p=0.011)$, stimulation place $(p=0.000)$, and stimulation level $(p=0.020)$ (similar results were found in Tang et al., 2006), while tinnitus suppression is unaffected by these same parameters. This suggests that neural mechanisms underlying loudness adaptation to electric stimuli are likely distinct from those underlying tinnitus adaptation.

Despite these differences, electric stimuli are able to elicit tinnitus suppression in a subset of this heterogeneous group of tinnitus subjects. Responder individuals who experienced tinnitus suppression here also exhibited a higher degree of adaptation to the electric stimuli, while Non-Responders achieved a lesser degree of adaptation to both their tinnitus and the electric stimulation percepts. This result supports the auditory gain hypothesis, in which increased central gain in response to reduced input from the periphery results in tinnitus (Salvi et al., 2000; Norena, 2011). If physiological mechanisms underlying loudness growth are mediated by cochlear and central non-linearities (Zeng and Shannon, 1994; Moore, 2004) and loudness adaptation may be mediated by a central feedback loop dependent on peripheral nerve activity (Tang et al., 2006), then our observation here of increased stimulus adaptation in Responder individuals may indicate that their tinnitus is such that the external stimulus, applied at the auditory periphery, can induce adaptation to the tinnitus as well. Accordingly, Non-Responders who exhibit less adaptation to both the stimulus and their tinnitus may have tinnitus which originates more centrally within the auditory system, making their tinnitus less susceptible to adaptation via peripheral stimulation.

\section{CLINICAL IMPLICATIONS}

We also compared an individual's tinnitus suppression outcome using our testing paradigm to their self-reported answer to the question "Has your tinnitus improved since cochlear implantation?" In Table 5, we see the patient's self-reported answer as "Helpful" or "Not Helpful" in rows, with any actual, validated effectiveness using the sounds in our study reported in columns as "Effective" or "Not Effective." Nearly half ( 6 of 13 subjects; $46.2 \%$ ) of the subjects inaccurately predicted actual effectiveness with our testing paradigm. Of these, two subjects predicted that their CI was helpful towards their tinnitus while not actually finding any effective sounds, while four subjects reported no benefit of their $\mathrm{CI}$, but found relief with our testing stimuli.

Table 5 | Expected versus actual effectiveness of tinnitus suppression through the $\mathrm{Cl}$.

\begin{tabular}{lllll}
\hline & & \multicolumn{3}{c}{ Research processor } \\
\cline { 3 - 5 } & & Effective & Not effective & Total \\
\hline $\begin{array}{l}\text { Own speech processor } \\
\text { (self-report) }\end{array}$ & Helpful & 5 & 2 & 7 \\
& Not helpful & 4 & 2 & 6 \\
& Total & 9 & 4 & 13
\end{tabular}


While this result could be explained by inaccurate reporting by the subjects of actual helpfulness of their CI, it seems less likely that subjects would be oblivious to a significant change to a bothersome percept and more likely that tinnitus suppression achieved using a CI with stimulation parameters ideal for speech understanding may be different from that using a CI optimized for tinnitus suppression. This effect is further clarified in Figure 6, which shows this relationship between speech recognition (using their own clinical speech processors) and tinnitus suppression (using research processors) for each subject, with Non-Responders in open diamonds and Responders in filled diamonds. The lack of correlation shown here $\left(R^{2}=0.02\right.$, $p=0.68$, Pearson correlation) suggests that optimal stimulation patterns may indeed be distinct for speech understanding and tinnitus suppression needs. Thus, in order to use a CI to manage tinnitus, the clinical speech processor should be programmed not only for speech perception but also by using stimulus parameters optimal for achieving tinnitus suppression in that particular patient.

Furthermore, the two subjects (S4 and S5) who reported that their CI was helpful but did not find relief with our stimuli did find relief from stimulation of the CI, albeit outside the context of our research stimuli. S4 found no relief with any of our 18 stimuli, but reported a "relaxing" and calming effect from the afternoon she got home from our testing until she woke up the next morning. The tinnitus in her implanted ear had disappeared from a "roar into a slight whistle," and the patient felt calm and relaxed with an "unexpected and unusual relief from tinnitus." She had experienced unrelenting tinnitus for 21 years. This was only the third time and by far the longest relief she had ever experienced from her tinnitus; the previous times had lasted only for minutes. This anecdotal report suggests a possible long-term effect of

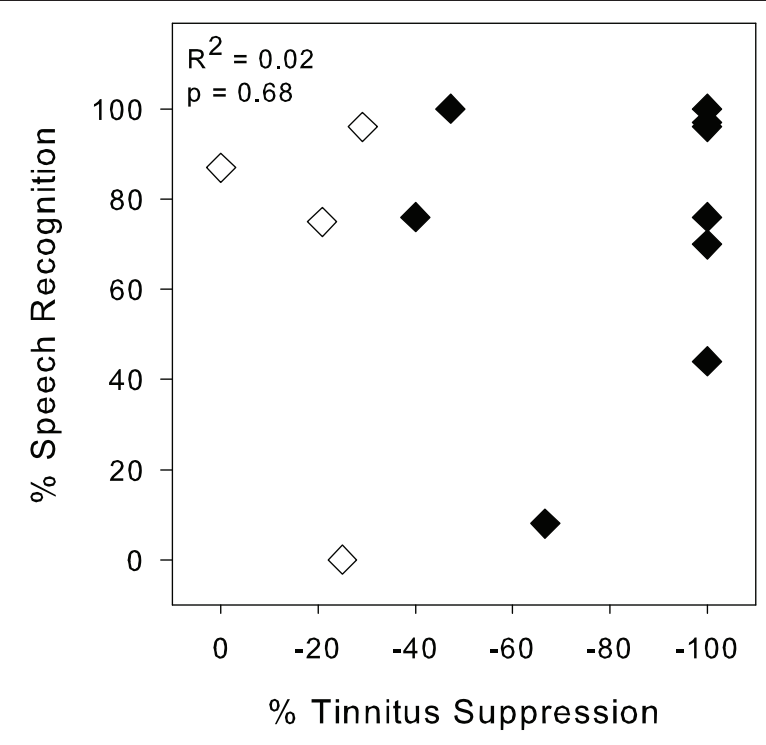

FIGURE 6 | Correlation of speech recognition with tinnitus suppression. The relationship between tinnitus suppression ( $x$-axis) and speech recognition scores ( $y$-axis) is shown for Responders (filled diamonds) and Non-Responders (open diamonds). electric stimulation on tinnitus that needs to be explored in the future.

S5 also experienced profound relief of her tinnitus with use of her CI, although her relief is immediate with activation of her implant. With her CIs inactivated, her tinnitus is regularly at a LR 7-8 ("loud but comfortable" to "maximal comfort") loudness. Activation of her CIs (she is a bilateral user) brings her tinnitus down nearly instantaneously to a LR 0 , or complete imperceptibility in environments with ambient noise. With CIs activated, her tinnitus does not exceed a LR 2 ("very soft") level even in a sound-attenuating booth; tinnitus suppression via electric cochlear stimulation is clearly effective for this patient. Some subjects may require multiple electrodes to be activated and/or dynamic stimulation to successfully achieve tinnitus suppression, as compared to our single-electrode, fixed stimulation tested here. Of note, $13 \%$ (30 of 227) of trials resulting in exacerbation of tinnitus were noted in the conditions tested here. This is consistent with exacerbation of tinnitus associated with CI use that has been noted in a small but notable number of patients (10\%) in other studies (Quaranta et al., 2004).

Lastly, we wanted to draw attention to any link between tinnitus and musical hallucinations in acquired deafness, with absence of evidence to suggest epilepsy or psychosis. One individual contacted us with tinnitus, but complaining especially of musical hallucinations. She was not enrolled in the study for unrelated reasons, but upon questioning, two enrolled subjects (one responder and one non-responder) reported musical hallucinations. Reported sounds varied from old Elvis songs to marching band and church bells; interestingly, no individual reported any lyrics or verbal hallucinations. Non-psychotic auditory hallucinations appear to be an underreported phenomenon associated with acquired deafness and especially in the elderly (Griffiths, 2000; Auffarth and Kropp, 2009).

\section{CONCLUSION}

Tinnitus suppression is possible via electrical stimulation of the cochlea in a subset of "Responder" subjects. A large variability between subjects, as well as the lack of a "most-effective" stimulus type (with respect to stimuli parameters: rate, place, or level), is indicative of the heterogeneity of the underlying tinnitus pathophysiology and individualized percept. These findings elucidate differences between two populations of individuals with tinnitus, Responders and Non-Responders, who not only respond to their tinnitus differently, but also exhibit different patterns of loudness adaptation to externally presented electric stimuli. These findings support the auditory gain hypothesis of tinnitus and suggest there may be characteristic differences in tinnitus generation between Responder and Non-Responder groups. It should be noted that while this study is to our knowledge the largest of its kind, the study size may nevertheless be relatively small given the grand diversity of tinnitus. Tinnitus suppression appears to be possible, but parameters of effective stimuli for tinnitus suppression may need to be customized for the individual.

\section{ACKNOWLEDGMENTS}

The authors would like to thank Essie Fine, Vanessa Rothholtz, and Hamid Djalilian for subject recruitment and data collection 
in two of the 13 subjects tested. The authors also thank Kelly M. Reavis and Hsin-I Yang for their insightful input and support during data analysis and production of the manuscript. They also are gratefully indebted to all the subjects who participated in this study for their patience, involvement, and time. This work was supported in part by grants from the American Tinnitus Association, National Institutes of Health (P30-DC008369 and T32-DC010775) and Medical Scientist Training Program (T32GM0862), as well as a fellowship from the Achievement Rewards for College Scientists Foundation.

\section{REFERENCES}

American Tinnitus Association. (2011). Support for Our Vets: One of Our Top Priorities. Portland: American Tinnitus Association. Available online: http://www.ata.org/actionalliance/support-for-veterans [Accessed 3 January 2011].

Amoodi, H. A., Mick, P. T., Shipp, D. B., Friesen, L. M., Nedzelski, J. M., Chen, J. M., and Lin, V. Y. (2012). Results with cochlear implantation in adults with speech recognition scores exceeding current criteria. Otol. Neurotol. 33, 6-12.

Arndt, S., Aschendorff, A., Laszig, R., Beck, R., Schild, C., Kroeger, S., Ihorst, G., and Wesarg, T. (2011). Comparison of pseudobinaural hearing to real binaural hearing rehabilitation after cochlear implantation in patients with unilateral deafness and tinnitus. Otol. Neurotol. 32, 39-47.

Auffarth, I. S., and Kropp, S. (2009). Musical hallucination in a patient after cochlear implantation. J. Neuropsychiatry Clin. Neurosci. 21, 230-231.

Axelsson, A., and Ringdahl, A. (1989). Tinnitus-a study of its prevalence and characteristics. Br. J. Audiol. 23, 53-62.

Baguley, D. M., and Atlas, M. D. (2007). Cochlear implants and tinnitus. Prog. Brain Res. 166, 347-355.

Balkany, T., Hodges, A., Menapace, C., Hazard, L., Driscoll, C., Gantz, B., Kelsall, D., Luxford, W., Mcmenomy, S., Neely, J. G., Peters, B., Pillsbury, H., Roberson, J., Schramm, D., Telian, S., Waltzman, S., Westerberg, B., and Payne, S. (2007). Nucleus Freedom North American clinical trial. Otolaryngol. Head Neck Surg. 136, 757-762.

Bovo, R., Ciorba, A., and Martini, A. (2010). Tinnitus and cochlear implants. Auris Nasus Larynx 38, 14-20.

Coles, R. R. A., Smith, P., and Davis, A. C. (1988). "The relationship between noise-induced hearing loss and tinnitus and its management," in 5th International Congress on Noise as a Public Health Problem, eds B. Berglund, J. Karlsson, and
T. Lindvall, (Stockholm: Swedish Council for Building Research).

Dauman, R., Tyler, R., and Aran, J. (1993). Intracochlear electrical tinnitus reduction. Acta Otolaryngol. 113, 291-295.

Eggermont, J. J., and Roberts, L. E. (2004). The neuroscience of tinnitus. Trends Neurosci. 27, 676-682.

Friesen, L. M., Shannon, R. V., Baskent, D., and Wang, X. (2001). Speech recognition in noise as a function of the number of spectral channels: comparison of acoustic hearing and cochlear implants. J. Acoust. Soc. Am. 110, 1150-1163.

Goodey, R. (2007). Tinnitus treatment: state of the art. Prog. Brain Res. 166, 237-246.

Griffiths, T. (2000). Musical hallucinosis in acquired deafness. Phenomenology and brain substrate. Brain 123 (Pt 10), 2065-2076.

Hazell, J., McKinney, C., and Aleksy, W. (1995). Mechanisms of tinnitus in profound deafness. Ann. Otol. Rhinol. Laryngol. Suppl. 166, 418-420.

House, J., and Brackmann, D. (1981). Tinnitus: surgical treatment. Ciba Found. Symp. 85, 204-216.

Ito, J., and Sakakihara, J. (1994). Tinnitus suppression by electrical stimulation of the cochlear wall and by cochlear implantation. Laryngoscope 104, 752-754.

Kaltenbach, J. A. (2011). Tinnitus: models and mechanisms. Hear. Res. 276, 52-60.

Miyamoto, R., Wynne, M., McKnight, C., and Bichey, B. (1997). Electrical suppression of tinnitus via cochlear implants. Int. Tinnitus J. 3, 35-38.

Moore, B. C. (2004). An Introduction to the Psychology of Hearing. London: Elsevier, Ltd.

Moore, B. C., Huss, M., Vickers, D. A., Glasberg, B. R., and Alcantara, J. I. (2000). A test for the diagnosis of dead regions in the cochlea. Br. J. Audiol. 34, 205-224.

Muhlnickel, W., Elbert, T., Taub, E., and Flor, H. (1998). Reorganization of auditory cortex in tinnitus. Proc. Natl. Acad. Sci. U.S.A. 95, 10340-10343.
Nilsson, M., Soli, S. D., and Sullivan, J. A. (1994). Development of the hearing in noise test for the measurement of speech reception thresholds in quiet and in noise. J. Acoust. Soc. Am. 95, 1085-1099.

Nondahl, D., Cruickshanks, K., Wiley, T., Klein, R., Klein, B., and Tweed, T. (2002). Prevalence and 5-year incidence of tinnitus among older adults: the epidemiology of hearing loss study. J. Am. Acad. Audiol. 13, 323-331.

Norena, A. J. (2011). An integrative model of tinnitus based on a central gain controlling neural sensitivity. Neurosci. Biobehav. Rev. 35, 1089-1109.

Pan, T., Tyler, R., Ji, H., Coelho, C., Gehringer, A., and Gogel, S. (2009). Changes in the tinnitus handicap questionnaire after cochlear implantation. Am. J. Audiol. 18, 144-151.

Quaranta, N., Wagstaff, S., and Baguley, D. (2004). Tinnitus and cochlear implantation. Int. J. Audiol. 43 245-251.

Roberts, L. E., Eggermont, J. J., Caspary, D. M., Shore, S. E., Melcher, J. R., and Kaltenbach, J. A. (2010). Ringing ears: the neuroscience of tinnitus. J. Neurosci. 30, 14972-14979.

Rubinstein, J., Wilson, B., Finley, C., and Abbas, P. (1999). Pseudospontaneous activity: stochastic independence of auditory nerve fibers with electrical stimulation. Hear. Res. 127 $108-118$.

Rubinstein, J. T., Tyler, R. S., Johnson, A., and Brown, C. J. (2003). Electrical suppression of tinnitus with high-rate pulse trains. Otol. Neurotol. 24, 478-485.

Ruckenstein, M. J., Hedgepeth, C., Rafter, K. O., Montes, M. L., and Bigelow, D. C. (2001). Tinnitus suppression in patients with cochlear implants. Otol. Neurotol. 22, 200-204.

Salvi, R. J., Wang, J., and Ding, D. (2000). Auditory plasticity and hyperactivity following cochlear damage. Hear. Res. 147, 261-274.

Shulman, A., Goldstein, B., and Strashun, A. M. (2009). Final common pathway for tinnitus: theoretical and clinical implications of neuroanatomical substrates. Int Tinnitus J. 15, 5-50.

Souliere, C. J., Kileny, P., Zwolan, T., and Kemink, J. (1992). Tinnitus suppression following cochlear implantation. A multifactorial investigation. Arch. Otolaryngol. Head Neck Surg. 118, 1291-1297.

Surr, R. K., Montgomery, A. A., and Mueller, H. G. (1985). Effect of amplification on tinnitus among new hearing aid users. Ear Hear. 6, 71-75.

Tang, Q., Liu, S., and Zeng, F. (2006). Loudness adaptation in acoustic and electric hearing. J. Assoc. Res. Otolaryngol. 7, 59-70.

Tyler, R., and Kelsay, D. (1990). Advantages and disadvantages reported by some of the better cochlear-implant patients. Am. J. Otol. 11, 282-289.

Tyler, R. S. (ed). (2000). Tinnitus Handbook. Clifton Park: Delmar, Cengage Learning.

UK Cochlear Implant Study Group. (2004). Criteria of candidacy for unilateral cochlear implantation in postlingually deafened adults I: theory and measures of effectiveness. Ear Hear. 25, 310-335.

van de Heyning, P., Vermeire, K., Diebl, M., Nopp, P., Anderson, I., and de Ridder, D. (2008). Incapacitating unilateral tinnitus in single-sided deafness treated by cochlear implantation. Ann. Otol. Rhinol. Laryngol. $117,645-652$.

Vernon, J. A. (2000). Masking of tinnitus through a cochlear implant. $J$. Am. Acad. Audiol. 11, 293-294.

Vernon, J. A., and Meikle, M. (2000). "Tinnitus Masking," in Tinnitus Handbook, ed R. Tyler (Clifton Park: Delmar, Cengage Learning), 464.

Wilson, B. S., Finley, C. C., Lawson, D. T., Wolford, R. D., Eddington, D. K., and Rabinowitz, W. M. (1991). Better speech recognition with cochlear implants. Nature 352, 236-238.

Zeng, F. G. (2004). Trends in cochlear implants. Trends Amplif. 8, 1-34.

Zeng, F. G., Rebscher, S., Harrison, W. V., Sun, X., and Feng, H. (2008). Cochlear implants: system design, integration and evaluation. 
IEEE Rev. Biomed. Eng. 1, $115-142$.

Zeng, F. G., and Shannon, R. V. (1994). Loudness-coding mechanisms inferred from electric stimulation of the human auditory system. Science 264, 564-566.

Zeng, F. G., Tang, Q., Dimitrijevic, A., Starr, A., Larky, J., and Blevins, N.
H. (2011). Tinnitus suppression by low-rate electric stimulation and its electrophysiological mechanisms.

Hear. Res. 277, 61-66.

Conflict of Interest Statement: The authors declare that the research was conducted in the absence of any commercial or financial relationships that could be construed as a potential conflict of interest.

Received: 10 January 2012; paper pending published: 25 January 2012; accepted: 13 March 2012; published online: 29 March 2012.

Citation: Chang JE and Zeng F (2012) Tinnitus suppression by electric stimulation of the auditory nerve.
Front. Syst. Neurosci. 6:19. doi: 10.3389/ fnsys.2012.00019

Copyright (๑) 2012 Chang and Zeng. This is an open-access article distributed under the terms of the Creative Commons Attribution Non Commercial License, which permits non-commercial use, distribution, and reproduction in other forums, provided the original authors and source are credited. 